\title{
A COMPOSIC̣ÃO CORPORAL, O VO2MÁX. E O ESTILO DE VIDA DE JOVENS ESTUDANTES DO ENSINO SUPERIOR
}

\author{
BODY COMPOSITION, VOZMÁX. AND THE LIFESTYLE \\ OF YOUNG STUDENTS OF THE HIGHER EDUCATION
}

\author{
AUTORES \\ João Mendes Leal ${ }^{1}$ \\ Tomás García Calvo ${ }^{2}$ \\ Pedro Antonio Sánchez Miguel ${ }^{2}$ \\ Ruperto Menayo Antúnez ${ }^{2}$ \\ Eduardo Cervelló Gimeno \\ 'Escola Superior de Educação de Beja (Portugal] \\ ${ }^{2}$ Universidade de Extremadura (Espanha) \\ ${ }^{3}$ Universidad Miguel Hernández de Elche \\ (Espanha)
}

A COMPOSIC̣ÃO CORPORAL, O VO2 MÁX. E O ESTILO DE VIDA DE JOVENS ESTUDANTES DO ENSINO SUPERIOR 4(4): 73-79

\section{PALAVRAS-CHAVE}

composição corporal; hábitos de vida; VO2 máx.

\section{KEYWORDS}

body composition; lifestyle, V02máx.

RESUMO

OBJECTIVO: Este estudo teve como propósito caracterizar os elementos da amostra relativamente à sua composição corporal, através do índice de massa corporal e da percentagem de massa gorda, à capacidade cardiorrespiratória, aos hábitos alimentares e de consumo de tabaco, índices de prática de actividade física e verificar se existiam relações estatisticamente significativas entre estas variáveis. MÉTODOS: 0 estudo decorreu em 2006 e 2007 e foi realizado no município de Beja, cidade do Sul de Portugal. Foi utilizada uma amostra constituída por 183 alunos que frequentavam no ano lectivo 2006/07 o $1^{\circ}$ ano dos cursos existentes numa instituição do ensino superior, com idades compreendidas entre 18 e 40 anos. Através de um questionário, da prova de Astrand e da técnica da bioimpedância eléctrica obtivemos os resultados que nos permitiram atingir os objectivos pretendidos.

Utilizámos o programa Bodygram para a obtenção dos dados relativos ao índice de massa corporal e à percentagem de massa gorda e o programa SPSS versão 14.0, para realizar a análise descritiva dos dados, uma análise de variância e ainda uma análise correlacional. RESULTADos: Verificámos que $62,8 \%$ dos elementos da amostra apresentavam valores de percentagem de massa gorda acima do recomendado e que $55,7 \%$ obtiveram valores de V02máx. muito baixos. Verificámos também que $61,7 \%$ não se consideravam sujeitos activos no que se refere à prática de actividade física regular, apresentando-se esta variável correlacionada com o V02máx. $(p=0,01)$. CONCLusões: Pudemos concluir que a maioria dos elementos da nossa amostra apresentava baixos índices de adesão à prática da actividade física, deficiente capacidade cardiorrespiratória e valores elevados no que se refere à percentagem de massa gorda. A prática da actividade física revelou-se correlacionada positivamente com a capacidade cardiorrespiratória. É necessária a implementação de campanhas mais eficazes de combate à obesidade e de sensibilização para a prática regular da actividade física e de bons hábitos alimentares.

\section{ABSTRACT}

OBJECTIVE: The purpose of this study was to characterize the subjects of the sample in what concerns their corporal composition, through the index of corporal mass and of the percentage of fat mass, their cardiorespiratory capacity, their eating and smoking habits, and indexes of practice of physical activity. It also aimed to verify if there were statistically relevant relationships between these variables. METHODS: The study was carried out in 2006 and 2007, in Beja, a city in the South of Portugal. The sample comprised 183 students attending the first year of all the courses of a higher education institution in the school year of 2006/ /07, with ages ranging from 18 to 40 years old. Through a questionnaire, the Astrand Test, and the technique of electric bioimpedance, we obtained the results that allowed us to reach the intended objectives.

We used the program Bodygram to obtain the data related to the index of corporal mass and the percentage of fat mass, and the program SPSS, version 14.0, to accomplish the descriptive analysis of the data, a variance analysis and also a correlational analysis. RESULTS: We found evidence that $62.8 \%$ of the subjects of the sample presented values of percentage of fat mass above the recommended limits and that $55.7 \%$ obtained very low values of V02max. We also found that $61.7 \%$ didn't consider themselves active subjects in what concerns the regular practice of physical activity, and that this variable was correlated with V02máx. $(p=0.01)$. CONCLusions: We could conclude that most of the subjects of our sample presented low indexes of physical activity, deficient cardiorespiratory capacity and high values in what concerns the percentage of fat mass. The practice of physical activity showed to be positively correlated with the cardiorespiratory capacity. It is necessary to implement more effective campaigns to combat obesity and to sensitize for the regular practice of physical activity and for good eating habits. 


\section{INTRODUC̣ÃO}

A sociedade tem vindo a alterar os seus comportamentos ao longo das últimas décadas como consequência da evolução científica e tecnológica, passando dum estilo de vida em que a actividade física era uma dominante para um outro em que o sedentarismo e as rotinas predominam.

Segundo Giddens ${ }^{11}$ (2000) "Os nossos corpos estão a ser invadidos pela influência da ciência e da tecnologia, dando origem a novos dilemas". A este propósito dizia ainda que o corpo é cada vez mais algo que temos de criar e não simplesmente aceitar.

Por outro lado, os baixos índices de participação em actividades físicas e desportivas em crianças, jovens e adultos, assim como os inadequados hábitos alimentares e de consumo de substâncias nocivas para a saúde, nomeadamente o tabaco e o álcool, são um motivo de preocupação permanente na sociedade em que vivemos.

Em virtude destes factos, algumas doenças, designadas hoje como crónicas não transmissíveis, têm evoluído até aos nossos dias, e que apesar do conhecimento das suas origens e de algum controlo possível, dificilmente conseguem ser eliminadas. A melhor forma de intervir nestes domínios será através da prevenção e neste aspecto, a actividade física e a alimentação parecem ser considerados como dois dos factores determinantes.

Embora, por vezes, a evidência dos resultados obtidos não seja tão convincente como a esperada, tendo em atenção factores como a duração do próprio estudo, a amostra utilizada e ainda um conjunto de variáveis difíceis de controlar, parece não restarem dúvidas sobre a importância de promover a actividade física, combatendo o sedentarismo, o qual se acentua à medida que se caminha na idade (Biddle et al. ${ }^{4}$ 2004).

Segundo Adams \& White ${ }^{2}$ (2003), "Um óptimo nível de actividade física pode atrasar ou prevenir o desenvolvimento de cardiopatias isquémicas, diabetes mellitus, obesidade, hipertensão arterial, cancro do cólon, osteoporose, ansiedade e depressão. Na Inglaterra as recomendações actuais para a maximização da saúde são de que todos os adultos deviam fazer trinta minutos ou mais de actividade física de intensidade moderada, todos os dias da semana, preferencialmente." Num estudo divulgado pelo Center for Disease Control and Prevention /National Center for Health Statistics (2002) foi dado destaque ao número elevado de mortes relacionadas com doenças cardiovasculares, destacando que $54 \%$ destas ocorriam por doenças cardíacas coronárias [54\%], e 18\% por enfartes cardíacos.

A prevalência do excesso de peso e da obesidade tem-se incrementado de forma significativa nas últimas décadas, constituindo um grave problema de saúde pública, tanto nos países desenvolvidos como nos países em desenvolvimento.

Segundo Keim et al. ${ }^{14}$ (2004) "a incidência do sobrepeso e da obesidade nos Estados Unidos da América e em todo o mundo, alcançou grandes proporções epidémicas. Para intervir eficazmente, os profissionais ligados à área dos cuidados para a saúde, necessitam de integrar a actividade física apropriada e suficiente na vida do indivíduo".

United States Departement of Health and Human Services ${ }^{26}$ (2003) referem igualmente que a obesidade crescente entre 1991 e
2001 foi a causa de morte de 280.000 a 325.000 americanos em cada ano. Revelam ainda que esta cresce de acordo com a idade num estudo apresentado com indivíduos com idades superiores a 18 anos.

Estudo realizado entre 2003-2005 e apresentado recentemente por Carmo et al $^{6}$ (2006), mostrava-nos que em Portugal 52,4\% dos sujeitos da amostra eram obesos ou tinham sobrepeso, sendo os valores destes, respectivamente de $13,8 \%$ e $38,6 \%$.

Num estudo realizado, Suenaga ${ }^{24}$ (2002), concluiu existir uma relação significativa entre a actividade física desenvolvida e o índice de V02máx encontrado e ainda relações significativas entre este índice e a percentagem de massa gorda e o índice de massa corporal.

Outro estudo realizado por Janczak et $\mathrm{al}^{13}$ (2002) comprovou-se a existência de uma relação significativa entre a captação máxima de oxigénio (VO2máx.] e a adiposidade. Foi estabelecida uma correlação negativa com a idade dos homens. Conclusões idênticas são referidas por Blair et $\mathrm{al}^{5}$ (2004) citando estudos realizados por King et al (1991) e Asikainen et al (2002).

A industrialização, a urbanização, o desenvolvimento económico e a globalização dos mercados, provocaram alterações profundas nos estilos de vida do cidadão comum, nomeadamente no que se refere, aos regimes alimentares, consumo de tabaco e sedentarismo.

Os alimentos e os produtos alimentícios converteram-se em produtos básicos fabricados e comercializados num mercado que se alterou $e$ ampliou, passando de uma origem essencialmente local, para uma de nivel mundial, com todas as implicações que daí advieram. 
Sendo a prática regular da actividade física um factor que poderá contribuir de forma significativa para um estilo de vida mais saudável, vários resultados têm demonstrado a manutenção de baixos índices de adesão da população em geral, sendo mais evidentes nos elementos do género feminino [Souza \& Duarte ${ }^{23}$, 2005).

0 modelo transteórico de Prochaska \& Diclemente ${ }^{20}$ (1983), contrariamente a outros modelos comportamentais, procura oferecer sugestões explícitas de como as pessoas podem ser ajudadas a mudar os seus comportamentos, considerando a existência de várias fases de mudança que reflectem a dimensão temporal na qual a mesma se revela.

Neste modelo são identificadas 5 fases de mudança no domínio da actividade física, de acordo com o comportamento do indivíduo ou da intenção manifestada relativamente à prática da actividade física, nomeadamente: pré-contemplativa, contemplativa, preparação, acção e manutenção as quais poderão não ser lineares, mas sim apresentando alguma ciclicidade de acordo com as intervenções que possam ocorrer (Marcus \& Lewis ${ }^{16}$, 2003).

ESTADIO DE PRÉ-CONTEMPLAC̣ÃO - 0 indivíduo não participa em qualquer actividade física nem tem intenção de o fazer futuramente;

ESTADIO CONTEMPLATIVO - 0 indivíduo não faz qualquer tipo de actividade física mas pensa fazê-lo nos próximos 6 meses;

ESTADIO DE PREPARAC̣̃̃o - Enquadram-se aqui indivíduos que não tendo ainda adquirido um novo comportamento, já realizam algumas pequenas acções que evidenciam alguma mudança;
ESTADIO DE ACC̄̃̃O - 0 indivíduo já realiza uma actividade física de forma regular mas há menos de 6 meses;

ESTADIO DE MANUTENC̣̃̃O - 0 indivíduo já assumiu um comportamento de prática de actividade física há mais de 6 meses e pensa continuar a fazê-lo.

Tendo em conta estes factos procurámos neste estudo caracterizar uma comunidade de estudantes do ensino superior relativamente à sua condição cardiorrespiratória, percentagem de massa gorda, índices de adesão à prática da actividade física, hábitos alimentares e de consumo de tabaco. Procurámos verificar também se existiam relações significativas entre estas variáveis e se o sexo se apresentava como variável discriminatória.

\section{MÉTODOS}

A amostra foi constituída por todos os estudantes que frequentavam o $1^{\circ}$ ano de vários cursos ministrados numa instituição do ensino superior em Portugal, o que correspondeu a um total de 183 elementos com idades compreendidas entre os dezoito e os quarenta anos, sendo vinte do género masculino e cento e sessenta e três do género feminino.

O período para a recolha de dados decorreu entre Setembro de 2006 e Março de 2007, sendo numa primeira fase obtidos os dados relacionados com a caracterização da amostra, hábitos de prática de actividade física, hábitos alimentares e de consumo de cigarros através de questionário construído e validado para o efeito e numa se- gunda fase realizadas provas para a obtenção de resultados relacionados com a capacidade cardiorrespiratória e a composição corporal. Foram utilizados como instrumentos para a recolha dos dados, para além do questionário já referido, uma balança para a obtenção do peso, um estadiómetro para medir a altura, um cicloergómetro de marca Monark, modelo 818E, para a realização do teste de Astrand-Ryhming e para cálculo do índice de massa corporal e da percentagem de massa gorda um equipamento específico de bioimpedância eléctrica de marca Akern, modelo Bia-101. $\mathrm{O}$ seu protocolo de medida consiste na fixação de dois eléctrodos emissores e dois eléctrodos receptores. Os eléctrodos emissores são colocados distalmente na superfície dorsal da mão e do pé, no plano das cabeças do terceiro metacarpo e do terceiro metatarso, respectivamente. Os eléctrodos receptores são colocados proximalmente também na mão e no pé, situando-se o primeiro no pulso, num plano imaginário de união das duas apófises estilóides e o segundo, na região dorsal da articulação da tíbio-társica, na linha imaginária de união da parte mais saliente dos dois maléolos. Posteriormente faz-se passar uma corrente de excitação muito baixa ( $800 \mu \mathrm{A}$ ) e de uma frequência constante $[50 \mathrm{kHz}]$, a qual nos permite medir a impedância corporal total. Através da utilização do equipamento referido obtêm-se duas medidas: a reactância $(R z)$ e a resistência total $(X c)$ que são introduzidas no programa Bodygram (software que acompanha o equipamento], para que possa ser feito o tratamento dos dados, possibilitando uma análise mais objectiva. 


\begin{tabular}{lcc}
\hline Estadios & Feminino & Masculino \\
\hline $\begin{array}{l}\text { Não faço nem tenho intenção de iniciar } \\
\text { uma actividade física nos próximos seis meses }\end{array}$ & $3,7 \%$ & $0 \%$ \\
\hline $\begin{array}{l}\text { Não faço, mas penso iniciar uma actividade física } \\
\text { nos próximos seis meses }\end{array}$ & $12,9 \%$ & $15 \%$ \\
\hline $\begin{array}{l}\text { Não sou fisicamente activo, mas tenho dado } \\
\text { pequenos passos no sentido de me tornar activo }\end{array}$ & $49,7 \%$ & $10 \%$ \\
\hline $\begin{array}{l}\text { Faço actividade física regularmente } \\
\text { há menos de seis meses }\end{array}$ & $16 \%$ & $10 \%$ \\
\hline $\begin{array}{l}\text { Faço actividade física regularmente } \\
\text { há mais de seis meses }\end{array}$ & $17,8 \%$ & $65 \%$ \\
\hline
\end{tabular}

TABELA1

Motivação para a prática da actividade física (modelo transteórico).
Para o tratamento dos dados recorremos a um conjunto de procedimentos, na sua maioria com recurso ao programa estatístico S.P.S.S. (Statistical Package for the Social Sciences] para o Windows, versão 14.0.

\section{RESULTADOS}

Seguidamente passamos a apresentar os principais resultados obtidos no estudo efectuado tendo como referência os objectivos definidos e as várias hipóteses formuladas.

No que se refere à prática de actividade física, concluímos que apenas $38,3 \%$ afirmava fazê-lo regularmente (3 vezes por semana no mínimo), sendo que destes, 15,3\% o fazia há menos de seis meses.
Os restantes não se consideravam sujeitos activos, havendo mesmo 3,3\% que afirmava, não realizar actividade física, nem o pretendia vir a fazer. De realçar que os valores dos elementos do género feminino são muito mais acentuados que os do género masculino.

Relativamente à percentagem de massa gorda, pudemos comprovar que a maioria dos elementos da amostra apresentava valores bem acima do que é considerado normal pela bibliografia científica específica, isto é, 62,8\% apresentavam valores acima dos $30 \%$ de massa gorda (tabela 2). De referir que todos estes sujeitos pertenciam ao género feminino, apresentando-se assim esta variável como discriminatória ( $p=0,000)$.

Também no que se refere à capacidade cardiorrespiratória, verificámos que a maioria dos elementos

\begin{tabular}{cccccc}
$\begin{array}{c}\text { Entre 0\% } \\
\text { e 9,9\% }\end{array}$ & $\begin{array}{c}\text { Entre 10\% } \\
\text { e 19,9\% }\end{array}$ & $\begin{array}{c}\text { Entre 20\% } \\
\text { e 29,9\% }\end{array}$ & $\begin{array}{c}\text { Entre 30\% } \\
\text { e 39,9\% }\end{array}$ & $\begin{array}{c}\text { Entre 40\% } \\
\text { e 49,9\% }\end{array}$ & + de 50\% \\
\hline $0 \%$ & $7,7 \%$ & $29,5 \%$ & $45,9 \%$ & $15,8 \%$ & $1,1 \%$ \\
\hline
\end{tabular}

TABELA?

Análise de frequência da percentagem da massa gorda. da amostra apresentava resultados de V02máx abaixo dos valores considerados como médios (American College of Sport Medicine ${ }^{3}$, 2000). Concluímos que $55,7 \%$, se situavam abaixo do percentil 30 e que destes, $31,7 \%$ se encontravam abaixo do percentil 10. Os elementos do sexo feminino apresentaram niveis inferiores, verificando-se diferenças estatisticamente significativas entre os dois grupos $(p=0,00)$. Os valores de V02máx apresentaram-se igualmente correlacionados com a percentagem de massa gorda, mais concretamente, elementos com valores mais elevados de percentagem de massa gorda apresentavam piores resultados de V02máx. ( $p=0,000$ ).

Relativamente ao consumo de tabaco, pudemos comprovar que $68,3 \%$ (125) dos sujeitos da amostra afirmaram não fumar qualquer cigarro por dia e que cerca de $5 \%$ (9) disseram que fumavam 20 ou mais cigarros. Dos restantes, 12\% (22) fumavam em média 5 cigarros, $8,74 \%$ (16) 10 cigarros e $6 \%$ (11) cerca de 15 cigarros diariamente.

Relativamente aos hábitos alimentares, os valores médios encontrados para qualquer dos nutrientes, enquadram-se dentro dos valores estimados no intervalo recomendado (proteínas entre 10\% e 15\%; carboidratos entre $50 \%$ e $65 \%$; lípidos entre 25\% e 35\%), no entanto, podemos destacar os valores relacionados com os lípidos e os carboidratos que se posicionam em sentido inverso face ao considerado como recomendável.

Enquanto nos carboidratos, 36,1\% dos elementos da amostra se situam acima dos valores recomendados, já no que se refere aos lípidos, 50,3\% encontram-se abaixo dos níveis aconselháveis. 
Contrariamente às nossas expectativas, a idade, em termos globais, não se revelou como variável discriminatória relativamente ao índice de massa corporal, à percentagem de massa gorda e ao índice de V02máx, o mesmo acontecendo no que se refere ao nível de adesão à prática da actividade física,

Fazendo uma análise comparativa grupo a grupo, sómente verificámos diferenças estatisticamente significativas no que se refere à percentagem de massa gorda entre o grupo com idades compreendidas entre os 21/25 anos e o grupo com 26/30 anos (Sig. $=0,019$ ). Procurámos ainda concluir sobre o carácter preditivo do índice de massa corporal e da idade relativamente à percentagem de massa gorda (tabela 4) e da percentagem de massa gorda e da idade no que se refere ao V02máx. (tabela 5).

Como podemos verificar, os valores obtidos indicaram-nos que os modelos se ajustavam à explicação das variáveis em estudo [sig = 000), não tendo a idade qualquer significado na mesma.

\section{DISCUSSÃO}

A prevalência de percentagens de massa gorda veio confirmar os resultados obtidos noutros estudos já referidos anteriormente pela International Obesity Task Force ${ }^{12}$ (2005), Carmo et al $^{6}$ (2006) e dos referidos por Seedo (1999) e Enkid (2000), citados por Ministério de Sanidad y Consumo y Ministério de Educación y Ciência ${ }^{18}$ (2006).

Em relação à capacidade cardiorrespiratória verificámos que os resultados confirmaram os obtidos noutros estudos, nomeadamente, os apresentados por Blair et al. ${ }^{5}$ (2004), assim como Smith \& Mitchell $^{22}, 2000$ e Janczak et al $^{13}$, 2002.

\begin{tabular}{lccc}
\hline & $\begin{array}{c}\text { Abaixo do } \\
\text { recomendado }\end{array}$ & $\begin{array}{c}\text { De acordo com } \\
\text { o recomendado }\end{array}$ & $\begin{array}{c}\text { Acima do } \\
\text { recomendado }\end{array}$ \\
\hline Proteínas & $2,2 \%$ & $91,3 \%$ & $6,6 \%$ \\
Carbohidratos & $2,2 \%$ & $61,7 \%$ & $36,1 \%$ \\
Lípidos & $50,3 \%$ & $44,8 \%$ & $4,9 \%$ \\
\hline
\end{tabular}

TABELA3

Nutrientes consumidos.

\begin{tabular}{ccccc}
\hline Model 1 & R Sqare & Beta & t & Sig \\
\hline BMI &, 436 &, 660 & 11,828 &, 000 \\
\hline a Predictors: BMl, idade & & &
\end{tabular}

Predictors: BMI, idade

TABELA4

Modelo preditivo da percentagem de massa gorda através do índice de massa corporal.

\begin{tabular}{rcccc}
\hline Model 1 & R Sqare & Beta & t & Sig \\
\hline PercMG & ,363 &,- 603 & $-10,159$ &, 000 \\
\hline PercMG, idade & & & & TABELA5 \\
& Modelo preditivo do V02máx. através da percentagem de massa gorda.
\end{tabular}

Relativamente aos índices de prática de actividade física os baixos níveis encontrados estão em consonância com os obtidos por National Center for Chronic Disease Prevention and Health Promotion ${ }^{19}$ (1999); Matos et al $^{17}$ (2001); Biddle et al $^{4}$ (2004); European Union ${ }^{8}$ (2004); Souza \& Duarte (2005), os quais puderam comprovar que a maioria dos jovens adolescentes e adultos tinham baixos índices de prática de actividade física. No entanto não verificámos que esta variável estivesse relacionada directamente com o índice de massa corporal e percentagem de massa gorda, à excepção feita aos grupos 3 (Não sou fisicamente activo, mas tenho dado pequenos passos no sentido de me tornar activo) e 5 (Faço actividade física regularmente há mais de seis meses]. Estes valores parecem contrariar os obtidos por Kyle et al $^{15}$ (2001) em que os indivíduos mais activos apresentavam valores significativamente inferiores relativamente à percentagem de massa gorda.

Apresentava-se, no entanto discriminatória relativamente ao nível de adesão à prática regular da actividade física, o que veio a confirmar os resultados obtidos por Suenaga ${ }^{24}$ (2002), Fernandez et al $^{9}$ (2005).

0 sexo revelou-se igualmente uma variável discriminatória relativamente aos índices de adesão à prática de actividade física, confirmando-se os resultados observados pelo National Center for Chronic Disease Prevention and Health Promotion $^{19}$ (1999); Matos et al ${ }^{17}$ (2001); Aaron et al' ${ }^{1}$, (2002); Souza \& Duarte ${ }^{23}$ (2005). Também confirmámos a sua relação com a percentagem de massa gorda, mostrando os elementos do sexo feminino piores resultados à semelhança do observado pelo Deurenberg et $\mathrm{al}^{7}$, (2000), mas contrariando os apresentados pela International 
Obesity TaskForce ${ }^{12}$ (2005) e Carmo et $a^{6}$, (2006), uma vez que nestes estudos os homens tinham valores superiores aos elementos do género feminino. Já no que se refere à condição cardiorrespiratória, esta é mais elevada nos elementos do género masculino à semelhança do já referido por Smith \& Mitchell ${ }^{22}$, 2000.

A idade, contrariamente ao esperado, não se revelou como variável discriminatória relativamente às variáveis índice de massa corporal, percentagem de massa gorda e índices de V02máx, o mesmo acontecendo no que se refere ao nível de adesão à prática da actividade física, contrariando outros resultados divulgados, nomeadamente o de Aaron et al ${ }^{1}$ (2002), o qual revelou que a idade parece influenciar os índices de prática, reduzindo esta à medida que se caminha na adolescência. Também National Center for Chronic Disease Prevention and Health Promotion ${ }^{19}$ (1999), Kyle et $\mathrm{al}^{15}$ (2001) e Torres et $\mathrm{al}^{25}$ (2000), puderam comprovar que indivíduos mais idosos teriam uma tendência para apresentar diferenças significativas relativamente ao peso, ao índice de massa corporal e à percentagem de massa gorda, fundamentalmente no que se refere aos sedentários.

Os hábitos alimentares e de consumo de tabaco, não se apresentaram correlacionados significativamente com o índice de massa corporal, a percentagem de massa gorda e o nível de V02máx. dos sujeitos que faziam parte da nossa amostra. De referir ainda, que os valores médios apresentados relativamente aos nutrientes consumidos indicavam que estávamos perante uma alimentação adequada, embora $36,1 \%$ dos elementos apresentassem indicadores de consumirem carboidratos em excesso e 50,3\% deles revelassem consumir lípidos abaixo do recomendado. Relativamente à percentagem de indivíduos que consumiam tabaco $[31,7 \%$ ) são valores um pouco superiores aos revelados pelo United States Departementof Health and Human Services $^{26}$, em 2003 [28,5\%] e pela Fundação Portuguesa de Cardiologia ${ }^{10}$ em 2006 (20\% a 26\%).

\section{CONCLUSÕES}

Tendo como referência os estadios definidos no modelo transteórico, concluímos que os sujeitos que constituíam a nossa amostra apresentavam baixos níveis de adesão à prática de actividade física.

$\mathrm{Na}$ sua maioria, os elementos da amostra apresentavam elevadas percentagens de massa gorda e uma deficiente capacidade cardiorrespiratória.

O nível de adesão à prática da actividade física revelou-se relacionado positivamente com a capacidade cardiorrespiratória mas não com o índice de massa corporal ou a percentagem de massa gorda.

Em termos globais, a idade não se apresentou como variável discriminatória relativamente à percentagem de massa gorda, índice de massa corporal e capacidade cardiorrespiratória.

Os hábitos alimentares e de consumo de tabaco não se apresentaram relacionados com a capacidade cardiorrespiratória, o índice de massa corporal e a percentagem de massa gorda.

Existem correlações estatisticamente significativas entre o índice de massa corporal, a percentagem de massa gorda e a capacidade cardiorrespiratória.

\section{CORRESPONDÊNCIA}

João Alberto Mendes Leal

Escola Superior de Educação de Beja

Rua Pedro Soares

7800-295 - BEJA - PORTUGAL

E-mail: jleal@eseb.ipbeja.pt

\section{REFERÊNCIAS}

1. Aaron D, Storti K, Robertson R, Kriska A \& LaPorte (2002); Longitudinal study of the number and choice of leisure time physical activities from mid to laté adolescence: implications for school curricula and community recreation programs; Archives Pediatrics \& Adolescent Medicine, 156(11): 1075-80.

2. Adams J \& White M (2003): Are activity promotion interventions based on the transtheorical model effective? A critical review. British Journal of Sports Medicine, 37, 106-114.

3. American College of Sport Medicine (2000): ACSM's guidelines for exercise testing and prescription, four edition; Philadelphia, Lippincott Williams \& Wilkins, Inc.

4. Biddle S, Gorely T, \& Stensel D (2004); Health-enhancing physical activity and sedentary behaviour in children and adolescents; Journal of Sports Sciences, 22, 679-701.

5. Blair S, LaMonte M \& Nichaman (2004): The evolution of physical activity recommendations: how much is enough?; American Journal Clinic Nutricional, 79 (suppl): 913-20.

6. Carmo I, Santos O, Camolas J, Vieira J, Carreira M, Medina, Reis L \& Galvão Teles A. (2006): Prevalence of obesity in Portugal; Obesity Review, The Internacional Association for the study of Obesity, 7: 233-237. 
7. Deurenberg $P$, Deurenberg-Yap M, Wang J, Lin F, Schmidt G (2000): Prediction of percentage body fat from anthropometry and bioelectrical impedance in Singaporean and Beijing Chinese; Asia Pacific Journal Clinic Nuticion, $\mathrm{n}$ ㅇ 9(2).

8. European Union (2004): Media Consulta International AG - Sportive activity more than once a week in www.eyes-2004.info [consultado a 24 de Maio de 2005).

9. Fernandez M, Vicente M, Montesinos G \& Rodríguez F (2005): Valoración de las mejoras provocadas en la capacidad aeróbica en mujeres sedentárias trás un programa de ejercicios; Revista Internacional de Medicina y Ciencias de la Actividad Física y del Deporte, nำ17 - ISSN: 1577-0354.

10. Fundação Portuguesa de Cardiologia (2006): Algumas reflexões sobre o tabaco,

http://cardiologia.browser.pt/Pri meiraPagina.aspx?ID_Conteudo= 21 [consultado a 15 de Junho de 2006).

11. Giddens A (2000): Sociologia. Lisboa, Fundação Calouste Gulbenkian. 12. International Obesity Task Force \& European Association for the Study of Obesity (2005): EU platform on diet, physical activity and health, Brussels, March 152005.

13. Janczak R, Cabric M, Krakowiak H, Jeka S. \& Bozilow W. (2002): Cardio-pulmonary performance and the level of body fat in men of various ages; Fizjoterapia Polska, vol. 2, Issue 2: 140-144.
14. Keim N, Blanton C \& Kretsch M (2004) America's obesity epidemic: measuring physical activity to promote an active lifestyle, Journal of the American Dietetic Association; 104 (9): 1398-409.

15. Kyle U, Gremion G, Genton L, Slosman D, Golay A \& Pichard C (2001): Physical activity and fatfree and fat mass by bioelectrical impedance in 3853 adults; Medicine Science Sports Exercise; 33(4): 576-84.

16. Marcus B \& Levis B (2003): Physical Activity and the Stages of Motivational Readiness for Change Model; President's Council on Physical Fitness and Sports, Series 4, 1 - March.

17. Matos M, Carvalhosa S \& Diniz J (2001); Actividade física e prática desportiva nos jovens portugueses, 4, 1, Lisboa, FMH/PEPT/GPT.

18. Ministério de Sanidad y Consumo y Ministério de Educación y Ciência [2006) in www.aesa.msc.es - programa PERSEO-JLO6.pdf.

19. National Center for Chronic Disease Prevention and Health Promotion (CDC) (1999); Adolescents and young adults fact sheets in physical and health-a report of the surgeon general.

20. Prochaska J \& Diclemente C (1983): Stages and processes of self-change in smoking: Towards and integrative model of change. Journal of Consulting and Clinical Psychology, 51, 390-395.
21. Sallis J (2000): age-related decline in physical activity: a synthesis of human animal studies; Medicine Science Sports Exercise, Sep; 32(9): 1598-600.

22. Smith M \& Mitchell J, (2000) Adaptaciones cardiorrespiratorias al entrenamiento in Resource manual for guidelines for exercise testing and prescription (traducción: Eduardo Iriarte Goni); BarceIona, Editorial Paidotribo.

23. Souza G \& Duarte M (2005): Estágios de mudança de comportamento relacionados à atividade física em adolescentes; Rev. Brás. Med. Esporte - Vol 11, No 2 Mar/Abr: 104-08.

24. Suenaga T (2002): Classification of physical activity and health related variable in men; Japanese Journal of Hygiene, vol. 57, Issue 2, May: 513-521.

25. Torres G, Carrasco L \& Medina $J$ (2000): Investigación sobre los motivos por los que los estudiantes universitarios practican deporte. El caso de la Universidad de Granada; Revista Motricidad - 6, 95-105.

26. United States Department of Health and Human Services (2003): Prevention makes common "cents"; United States Department of Health and Human Services.

27. Vieira A, Alvarez M, Martins V, Sichieri R \& Veiga G (2006). Accuracy of different body mass index reference values to predict body fat in adolescents; Cadernos de Saúde Pública, Rio de Janeiro, 22(8): 1681-1690. 